\title{
Management of Extremely Long Sinus Pauses in a Child with Primitive Neuroectodermal Tumor and Apparent Life Threatening Events: A Case Report
}

\author{
Nikolaos G Eleftherakis* and Nikolaos D Andreou
}

Department of Pediatric Cardiology, 'Agia Sofia' Children's Hospital, Athens, Greece

\begin{abstract}
A male child, three years old, with primitive neuroectodermal tumor (PNET) was admitted to our department because of episodes of apnea with syncope. Twenty four hr ambulatory electrocardiographic monitoring revealed extremely long sinus pauses, the longest being thirty four seconds, followed by sinus tachycardia. Permanent pacing reduced the frequency, duration and severity of episodes.
\end{abstract}

Keywords: Apparent Life Threatening Event (ALTE); Oculogyration; Mortality rate

\section{Introduction}

Definition of Apparent Life Threatening Event (ALTE) was given by the National Institutes of Health Conference on Infantile Apnea and Home Monitoring in 1986: episode that is frightening to the observer that is characterized by some combination of apnea (central or occasionally obstructive), color change (usually cyanotic or pallid but occasionally erythematous or plethoric), marked change in muscle tone (usually marked limpness), choking or gagging. In some cases, the observer fears that the infant has died [1].

In an effort to prevent sudden death in infants with ALTE, home monitoring is advised and parents have been trained in cardiopulmonary resuscitation techniques. It has been demonstrated though that these measures have not reduced the mortality rate [2]. Major concern of the physician is to resolve further events, if possible, by treating the cause, once diagnosis is made. Purpose of the case we present is to demonstrate that permanent pacing is a symptomatic management option when extremely long sinus pauses appear during ALTE.

\section{Case Presentation}

We describe the case of a three year old boy with PNET of the cervical part of the spinal cord, who was admitted to our department because of apneic syncopal episodes the past six months (ALTE). He had undergone surgical procedure for the tumor of the central nervous system and was on maintenance experimental chemotherapy only. $\mathrm{He}$ suffered postoperative residual left hemiplegia and right hemiparesis.

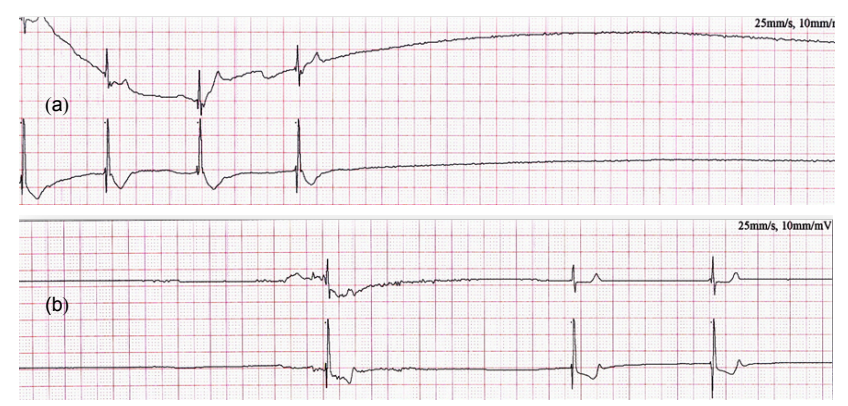

Figure 1: Cardiac pauses during apnea. They start with slow junctiona rhythm (a) and finish with junctional escape rhythm (b) followed by sinus tachycardia.
After an episode of syncope he was admitted to the Intensive Care Unit and on exit he had tracheostomy, kept since then. He fed through a permanent gastrostomy catheter. He had two central indwelling lines, one in right internal jugular vein for drainage of the cerebrospinal fluid (ventriculocaval shunt) and another, Hickman catheter, in superior vena cava. The parents reported a history of breath holding with rigidity of right upper and lower limb, pallor with perioral cyanosis and upward oculogyration, lasting three or four minutes. There was no clear correlation between the tumor and the events. Clinical examination of the cardiovascular system was unremarkable. Resting electrocardiogram was normal and echocardiographic examination was normal as well. Twenty four hour Holter monitoring of the cardiac rhythm revealed sinus pauses during the episodes, followed by sinus tachycardia. The longer pause lasted thirty four seconds. In view of

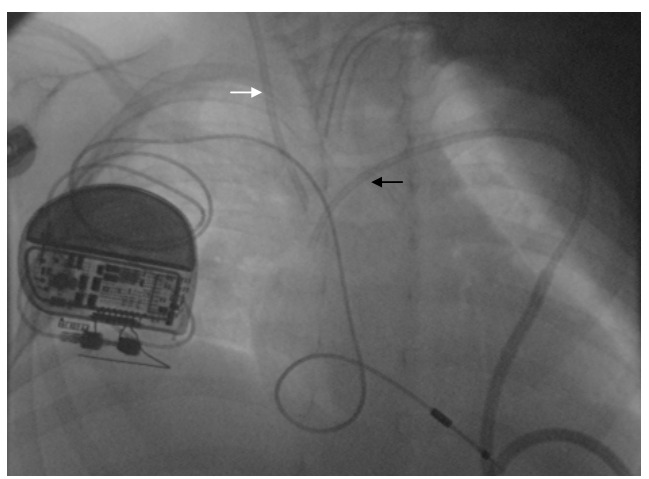

Figure 2: Pacemaker and ventricular lead after implantation. Illustrated also the catheter in right internal jugular vein (white arrow) for drainage of the cerebrospinal fluid and Hickman catheter, in superior vena cava (black arrow).

*Corresponding author: Nikolaos G Eleftherakis, Department of Pediatric Cardiology, Agia Sofia Children's Hospital, Thivon and Papadiamantopoulou St, 11527 Goudi, Athens, Greece, Tel: 00302107467105; Fax: 00302107797649; E-mail: ngeleftherakis@hotmail.com

Received June 11, 2014; Accepted July 18, 2014; Published July 21, 2014

Citation: Eleftherakis NG, Andreou ND (2014) Management of Extremely Long Sinus Pauses in a Child with Primitive Neuroectodermal Tumor and Apparent Life Threatening Events: A Case Report. J Clin Case Rep 4: 385. doi:10.4172/21657920.1000385

Copyright: (c) 2014 Eleftherakis NG, et al. This is an open-access article distributed under the terms of the Creative Commons Attribution License, which permits unrestricted use, distribution, and reproduction in any medium, provided the original author and source are credited. 
these findings a Permanent Magnetic Resonance Imaging (MRI) Safe VVI R Pacemaker was implanted. The ventricular lead was inserted through the right subclavian vein and was also MRI compatible. Twenty four hour ambulatory monitoring of the cardiac rhythm after implantation revealed no pauses. Symptoms of the child improved and frequency, duration and severity of episodes were reduced.

\section{Discussion}

Although precise incidence of ALTE is not known, the reported incidence is $0.05 \%$ to $6 \%$. They occur in children younger than one year, mostly in infants younger than ten weeks. They are more common in males than in females. Infants older than two years with recurrent episodes are more likely to have significant disorders [3]. There is a wide range of diagnoses reported as a cause of ALTE. Gastrooesophageal reflux disease is the commonest [4]. Seizure is a cause that can be established by certain diagnostic tools and is well documented that apnea may be a manifestation of seizures in infancy [5].

Primary cardiac causes are rare but sinus bradycardia is a common denominator of the events. Treatment is controversial because relation between ALTE and sudden infant death is not clear [6]. Wide QT Dispersion has been reported in infants with ALTE. This parameter is associated with increased risk of sudden death in heart failure and increased risk of ventricular fibrillation in acute myocardial infarction [7].

ALTE is a management dilemma as between events children are in good condition. The case we present illustrates that interventional management of selected cases of children with ALTE can be beneficial.

It has been reported that surgical management of children with underlying cardiac disorder has eliminated the episodes [8]. In the case we present, pacing was considered necessary because of the extremely long duration of the sinus pauses. We presumed that maintaining cardiac output during the events could palliate symptoms and protect the child from cardiac arrest. Therapeutic results support our initial hypothesis. MRI compatible pacemakers and pacing leads have been introduced into clinical practice recently. They should be implanted in any child with increased likelihood of MRI assessment afterwards.

\section{References}

1. (1987) National Institutes of Health Consensus Development Conference on Infantile Apnea and Home Monitoring, Sept 29 to Oct 1, 1986. Pediatrics 79: 292-299.

2. Committee on Fetus and Newborn American Academy of Pediatrics. (2003) Apnea, sudden infant death syndrome, and home monitoring. Pediatrics 111: 914-917.

3. Hall KL, Zalman B (2005) Evaluation and management of apparent lifethreatening events in children. Am Fam Physician 71: 2301-2308.

4. Tirosh E, Jaffe M (1996) Apnea of infancy, seizures, and gastroesophageal reflux: an important but infrequent association. J Child Neurol 11: 98-100.

5. McGovern MC, Smith MB (2004) Causes of apparent life threatening events in infants: a systematic review. Arch Dis Child 89: 1043-1048.

6. Esani N, Hodgman JE, Ehsani N, Hoppenbrouwers T (2008) Apparent lifethreatening events and sudden infant death syndrome: comparison of risk factors. J Pediatr 152: 365-370.

7. Goldhammer El, Zaid G, Tal V, Jaffe M, Abinader EG (2002) QT dispersion in infants with apparent life-threatening events syndrome. Pediatr Cardiol 23 605-607.

8. Martin MH, Perry SB, Prochazka JV, Hanley FL, Silverman NH (2010) Surgical management of life threatening events caused by intermittent aortic insufficiency in a native valve: case report. J Cardiothorac Surg 5: 94. 